\title{
An unusual case of idiopathic inflammatory myopathy presenting with organizing pneumonia as cavitary lesions
}

\begin{abstract}
A young woman presented with gradually progressive breathlessness, cough and muscle weakness for one and a half year. Her chest radiograph showed multiple, bilateral thin-walled cavitary lesions. Her serum was found to be positive for anti-Jo1 antibodies on serology. Histopathology of lung lesions was suggestive of organizing pneumonia. The muscle biopsy demonstrated a myopathic pattern. On the basis of these findings she was diagnosed to be the case of idiopathic inflammatory myopathy. Her condition improved significantly after treatment with steroids.
\end{abstract}

Key words: ILD, IIM, organizing pneumonia

\section{Introduction}

Inflammatory idiopathic myopathies (IIM) are a group of uncommon autoimmune disorders [1]. The clinical presentation includes proximal skeletal muscle weakness, raised muscle enzymes (e.g. creatine kinase, CK) and extramuscular organ involvement, most frequently the lungs, resulting in interstitial lung disease (ILD). Organizing pneumonia is a rare form of ILD in IIM. Here, we present an unusual case of idiopathic inflammatory myopathy presenting with organizing pneumonia as cavitary lesions.

\section{Material and methods}

A 38-year-old housewife presented with breathlessness associated with dry cough and low-grade, intermittent fever. The breathlessness gradually progressed from mMRC grade 1 to grade 4 , over a period of one and half years. There were no complaints indicative of a connective tissue disorder such as joint pain, Raynaud's phenomenon, skin rash, photosensitivity, dryness of the eyes or mouth, loss of appetite or weight. She denied any exposure to tobacco smoke, drugs (like nitrofurantoin, methotrexate, bleomycin etc.) or having pets in the house. There was no past history of similar complaints. Family history of any significant illness could not have been elicited.

On physical examination she was conscious, cooperative, and well oriented to time and place. Her pulse rate was $126 / \mathrm{min}$, regular; respiratory rate was $24 / \mathrm{min}$ and oxygen saturation was $88 \%$ while breathing room air. An erythematous and itchy rash was also observed over the face about which patient had not complained initially (Figure 1). On auscultation of the chest, bilateral end-inspiratory crackles were heard. Chest X-ray demonstrated cyst-like opacities in the left lower zone (Figure 2). Computed tomography scan of the chest showed bilaterally multiple, round to irregular, thin-walled cavities, which were in communication with the segmental bronchi. Centrilobular nodules with a tree-in-bud pattern were also observed in the posterior segment of the right upper lobe, lateral segment of the middle lobe, superior segment of the right lower lobe and all segments of the left lower lobe. No significant mediastinal or hilar lymphadenopathy was noticed

Address for correspondence: Jagdish Chander Suri, Medeor JCS Institute of Pulmonary, Critical Care \& Sleep Medicine, $33-34$ Qutab Institutional Area, 110016 New Delhi, India; e-mail: docjcsuri@gmail.com

DOI: 10.5603/ARM.2019.0041

Received: 28.01.2019

Copyright (C) 2019 PTChP

ISSN 2451-4934 
(Figure 3). Her arterial blood gas analysis was suggestive of hypoxic respiratory failure with wide alveolar-arterial oxygen gradient and respiratory alkalosis. The hemoglobin, total and differential leucocyte counts, liver and renal function tests were within normal limits. Spirometry suggested a mixed obstructive and restrictive ventilatory pattern with values of FEV1/FVC 71.19\% (less

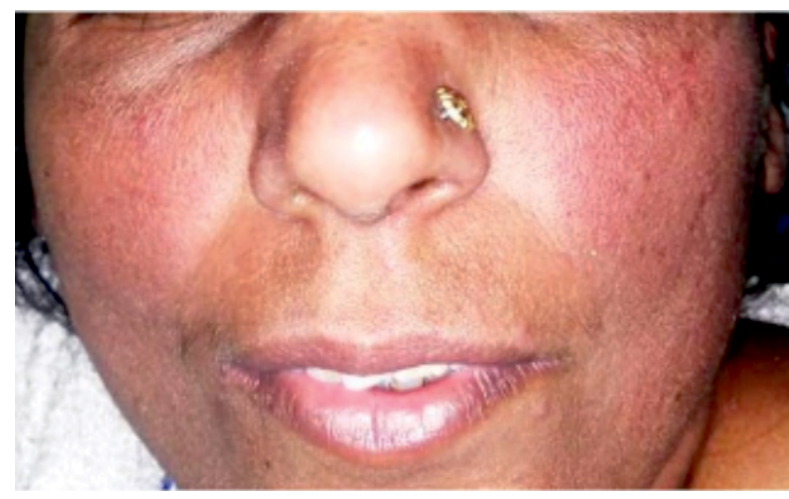

Figure 1. Bilateral malar rash

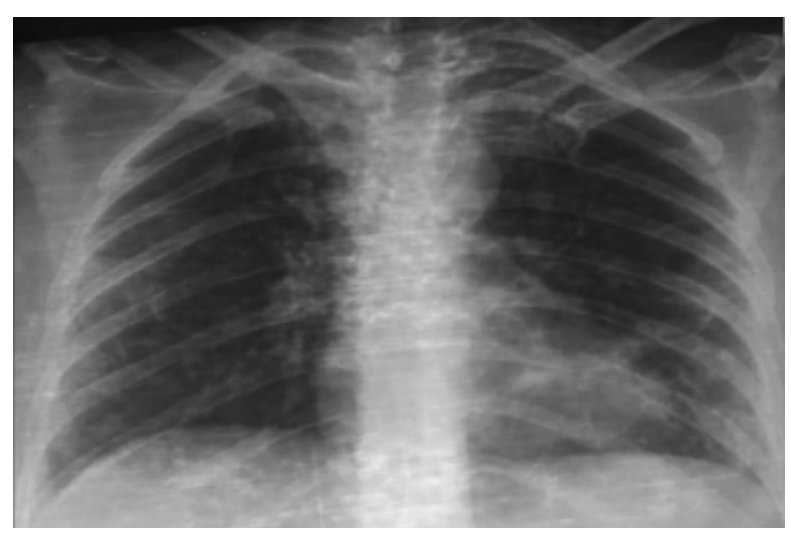

Figure 2. Chest X-ray (PA view) showing cavitary lesions in the left lower zone than lower limit of normal), FVC $1.92 \mathrm{~L}$ (62\% of predicted value) and $\mathrm{FEV}_{1} 1.37 \mathrm{~L}(52 \%$ of predicted value). Examination of both sputum and bronchoalveolar lavage (BAL) fluid was negative for acid-fast bacilli (AFB) on direct smear and culture. The cartridge-based nucleic acid amplification test (CB-NAAT) was also negative for tuberculosis (Xpert MTB/RIF). No atypical cells were seen. Although it is known that in DPLD, histological support for a specific diagnosis can be obtained using transbronchial lung biopsy (TBLB) in 29-79\% of cases only [2], since we do not have facilities for surgical lung biopsy in our set-up, we went ahead with transbronchial lung biopsy. This was done with the intent that if TBLB is negative then we will shift the patient to a center where such facility is available. The histopathology of transbronchial lung biopsy specimen demonstrated a moderate interstitial lymphoid infiltrate with organizing pattern of nodules and remodeling near bronchovascular regions. The nodules had spindle cells having myxoid stroma in the form of intra-alveolar buds and without any evidence of granuloma formation, atypical cells or fungal organisms consistent with organizing pneumonia. This pathology finding was confirmed by two pathologists independently. Based on these initial clinical and laboratory parameters, we arrived at the diagnosis of organizing pneumonia with multiple cavitary lesions.

In order to differentiate it from idiopathic organizing pneumonia and find a secondary cause, meticulous history and systemic examination was performed. On detailed probing, the patient reported that she had experienced difficulty in getting up from the squatting position and also while combing her hair for about a week. On physical examination, there was bilateral proximal muscle weakness in the upper and lower limbs (motor

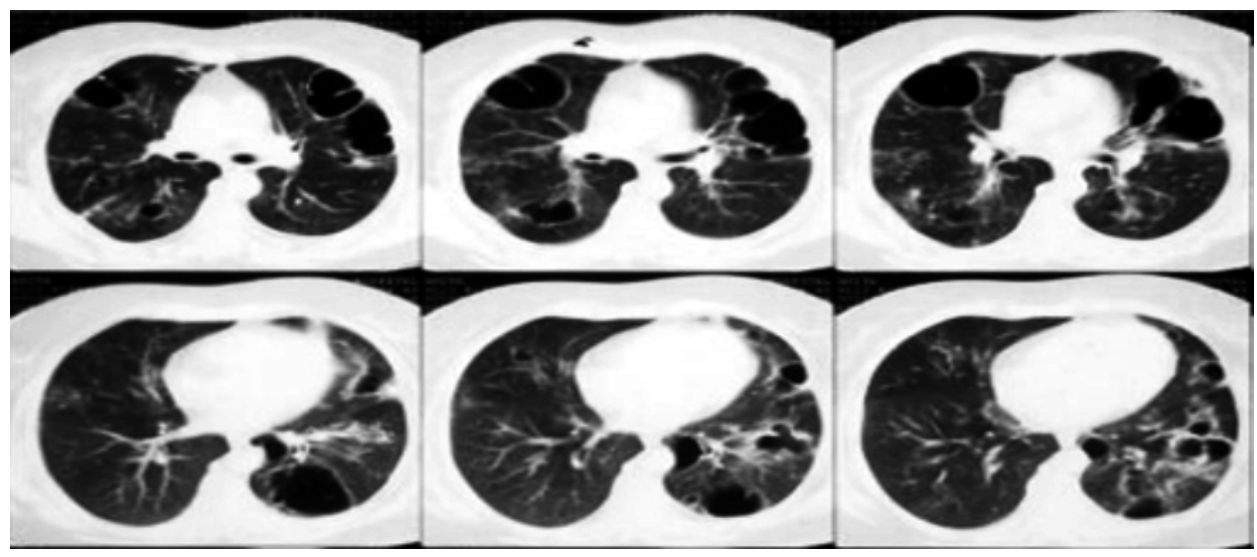

Figure 3. Computed tomography scan showing bilateral cavitary lesion 
power of 4/5, normal muscular tone), with normal deep tendon reflexes and sensory system. Electromyography (EMG) and nerve conduction velocity (NCV) studies were suggestive of a myopathic pattern. The level of serum creatinine phosphokinase (CPK) was elevated to $700 \mathrm{U} / \mathrm{L}$ (normal 38-176 U/L in females). Anti-Jo-1 antibody test was positive, suggesting an inflammatory myopathy. Other autoimmune markers like anti-nuclear antibodies (ANA), anti-ds DNA, anti-Ro52 recombinant, anti -SS-B antibody, anti-U1-RNP/Sm, anti-La antibody, anti-cardiolipin antibody, rheumatoid factor and anti-CCP antibody, anti-phospholipid antibodies (APLA), were negative. The direct Coombs' test was also negative. Complement C3 level was $87 \mathrm{mg} / \mathrm{dL}$ (normal range $90-180 \mathrm{mg} / \mathrm{dL}$ ), and C4 level was $21 \mathrm{mg} / \mathrm{dL}$ (normal range 15-57 mg/dL). Muscle biopsy showed the presence of endomysial inflammation.

The history of muscle weakness, laboratory measurements of CPK and anti-Jo1 antibody along with the muscle biopsy report were consistent with the diagnosis of idiopathic inflammatory myopathy with polymyositis. This was in accordance with the American College of Rheumatology and European League Against Rheumatism (ACR/EULAR) diagnostic criteria for adult and juvenile idiopathic inflammatory myopathies and their major subgroups 2017 [1]. She was subsequently treated with oral prednisolone $(1 \mathrm{mg} / \mathrm{kg}$ body weight) for a period of four weeks. A follow-up visit after 4 weeks showed clinical improvement in her symptoms, ABG and CPK levels.

\section{Discussion}

Organizing pneumonia is a rare lung disease that is characterized by an inflammatory involvement of bronchioles and alveoli, which responds very well to corticosteroids. It usually presents with a subacute course of fever or flu-like illness associated with cough, weight loss and mild dyspnea. It is defined histopathologically by the presence of intra-alveolar buds of granulation tissue consisting of an admixture of myofibroblasts and connective tissue [3]. It has been reported to occur in association with a variety of conditions like: infection (lung abscess, necrotizing pneumonia, septic pulmonary emboli, fungal infections, parasitic diseases, viral infection), malignancy (primary lung cancer, most commonly squamous cell carcinoma, others being primary or secondary pulmonary lymphoma, pulmonary metastases), others being rheumatoid nodules developing central necrosis, granulomatosis with polyangiitis (GPA), ankylosing spondylitis, primary pulmona- ry amyloidosis, eosinophilic granulomatosis with polyangiitis, sarcoidosis and systemic lupus erythematosus. It may be termed as idiopathic in case no causative factor is identified [4]. Our patient's BAL fluid for infectious causes was negative, no malignant cells were seen. She had Anti-Jo-1 Ab-positive, and TBLB was suggestive of organizing pneumonia. This antibody is positive in cases of idiopathic inflammatory myopathy (IIM) which is a group of immune-mediated disorders that are sub-categorized into polymyositis (PM), dermatomyositis (DM) and inclusion body myositis (IBM) [5]. These disorders can be associated with a range of myositis-specific auto-antibodies to aminoacyl t-RNA synthetases, signal recognition particles and nuclear helicase Mi-2. The patient described in the report had anti-Jo-1 antibodies directed against the anti-histidyl-tRNA synthetase. This antibody is usually detectable in 15-30\% of myositis patients and more often in the polymyositis ones [5]. Idiopathic inflammatory myopathy is a relatively rare disorder with prevalence of 0.5-8.4 cases per million population [6].

Interstitial lung disease (ILD) has been reported in $20-80 \%$ of cases of IIM [7-10]. Various forms of ILD have been described, including non-specific interstitial pneumonitis (NSIP), usual interstitial pneumonia (UIP), organizing pneumonia and diffuse alveolar damage (DAD). Organizing pneumonia is a rare form of ILD in IIM. In the two case series of IIM, ILD was reported to be present in 2 out of 36 patients in the first [11] and 1 out of 22 patients in the second case series [12]. Organizing pneumonia can present with varied radiological features. The radiological features described in various reports are focal lesion, nodules, bronchocentric picture, linear band-like shadows, perilobular lesions, cavitary and ring-like shadows, and progressive fibrotic opacities [13]. Mehrian et al. have reported that HRCT features like lower lobe pneumonia, sub-pleural reticulation, non-septal linear opacities, bronchial dilatation and reverse halo sign were more likely to occur in organizing pneumonia [14]. The unusual findings of organizing pneumonia presenting as cavitary lesions in our case have also been reported in three other case reports [15-17]. Organizing pneumonia was confirmed by surgical biopsy in these cases. It has been suggested that pathogenesis of a cavity formation in these cases could be as a consequence of inflammatory injury to the lung with focal areas of necrosis within organizing pneumonia [3]. Additionally, it may be likely that some of these cavities are secondary to pneumatoceles. 


\section{Conclusion}

Our patient presented with an uncommon manifestation of cavitary form of organizing pneumonia that was secondary to an inflammatory disorder. Also, the symptoms of her primary disorder presented much later than the ILD. This rare presentation underscores the importance of a thorough evaluation of any cavitary lesion before labelling it as idiopathic organizing pneumonia.

\section{Conflict of interest}

The authors declare no conflict of interest.

\section{References:}

1. Lundberg IE, Tjärnlund A, Bottai M, et al. 2017 European League Against Rheumatism/American College of Rheumatology classification criteria for adult and juvenile idiopathic inflammatory myopathies and their major subgroups. Ann Rheum Dis. 2017; 76(12): 1955-1964, doi: 10.1136/annrheumdis-2017-211468.

2. Wells AU, Hirani N, Thoracic B, et al. Interstitial lung disease guideline: the British Thoracic Society in collaboration with the Thoracic Society of Australia and New Zealand and the Irish Thoracic Society. 1999.

3. Cordier JF. Cavitary bronchiolitis obliterans organizing pneumonia. Eur Respir J. 1995; 8(11): 1822-1823, indexed in Pubmed: 8620945.

4. Naidich D, Machnicki S, Brown KK, Husta B, Ryu J, Sarosi G, et al. AC SC. Chest. 2018.

5. Miller ML, Flaherty KR, Curtis MR. Interstitial lung disease in dermatomyositis and polymyositis: Clinical manifestations and diagnosis. 2018(Dm): 5-12.

6. Kalluri M, Oddis CV. Pulmonary manifestations of the idiopathic inflammatory myopathies. Clin Chest Med. 2010; 31(3): 501-512, doi: 10.1016/j.ccm.2010.05.008, indexed in Pubmed: 20692542 .
7. Fathi M, Vikgren J, Boijsen M, et al. Interstitial lung disease in polymyositis and dermatomyositis: longitudinal evaluation by pulmonary function and radiology. Arthritis Rheum. 2008; 59(5): 677-685, doi: 10.1002/art.23571, indexed in Pubmed: 18438901.

8. Love LA, Leff RL, Fraser DD, et al. A new approach to the classification of idiopathic inflammatory myopathy: myositis-specific autoantibodies define useful homogeneous patient groups. Medicine (Baltimore). 1991; 70(6): 360-374, doi: 10.1097/00005792-199111000-00002, indexed in Pubmed 1659647.

9. Marie I, Hachulla E, Chérin P, et al. Interstitial lung disease in polymyositis and dermatomyositis. Arthritis Rheum. 2002; 47(6): 614-622, doi: 10.1002/art.10794, indexed in Pubmed: 12522835.

10. Bartosiewicz M, Siemion-Szcześniak I, Sobiecka M, et al. [Interstitial lung disease in patients with polymyositis and dermatomyositis--report of three cases]. Pneumonol Alergol Pol. 2013; 81(6): 556-566, indexed in Pubmed: 24142786.

11. Douglas WW, Tazelaar HD, Hartman TE, et al. Polymyositis-dermatomyositis-associated interstitial lung disease. Am J Respir Crit Care Med. 2001; 164(7): 1182-1185, doi: 10.1164/ ajrccm.164.7.2103110, indexed in Pubmed: 11673206.

12. Koreeda Y, Higashimoto I, Yamamoto M, et al. Clinical and pathological findings of interstitial lung disease patients with anti-aminoacyl-tRNA synthetase autoantibodies. Intern Med. 2010; 49(5): 361-369, doi: 10.2169/internalmedicine.49.2889, indexed in Pubmed: 20190466.

13. Organizing pneumonia: the many morphological faces. Eur Radiol. . 2002 ; 12(6): 1486-1496, doi: 10.1007/s00330-001-1211-3.

14. Mehrian P, Doroudinia A, Rashti A, et al. High-resolution computed tomography findings in chronic eosinophilic vs. cryptogenic organising pneumonia. Int J Tuberc Lung Dis. 2017; 21(11): 1181-1186, doi: 10.5588/ijtld.16.0723, indexed in Pubmed: 29037300.

15. Haro M, Vizcaya M, Texidó A, et al. Idiopathic bronchiolitis obliterans organizing pneumonia with multiple cavitary lung nodules. Eur Respir J. 1995; 8(11): 1975-1977.

16. Heller I, Biner S, Isakov A, et al. TB or not TB: cavitary bronchiolitis obliterans organizing pneumonia mimicking pulmonary tuberculosis. Chest. 2001; 120(2): 674-678, doi: 10.1378/ chest.120.2.674, indexed in Pubmed: 11502679.

17. Voloudaki AE, Bouros DE, Froudarakis ME, et al. CT features in two cases of bronchiolitis obliterans organizing pneumonia (BOOP). Acta Radiol. 1996; 37: 889-892, doi: 10.1036/10978542.yb081110. 